\title{
SINAIS CLIMÁTICOS EM ANÉIS DE CRESCIMENTO DE Cedrela fissilis EM DIFERENTES TIPOLOGIAS DE FLORESTAS OMBRÓFILAS DO SUL DO BRASIL
}

\author{
Fernando Andreacci ${ }^{1}$, Paulo Cesar Botosso ${ }^{2}$, Franklin Galvão ${ }^{3}$ \\ ${ }^{1}$ Biólogo, M. Sc., Depto. de Botânica, UFPR, Curitiba, PR, Brasil - fandreacci@gmail.com \\ ${ }^{2}$ Eng. Florestal, Dr., EMBRAPA Florestas, Colombo, PR, Brasil - paulo.botosso@gmail.com \\ ${ }^{3}$ Eng. Florestal, Dr., Depto. de Ciências Florestais, UFPR, Curitiba, PR, Brasil - fgalvao@ufpr.br
}

Recebido para publicação: 29/05/2012 - Aceito para publicação: 02/12/2013

\begin{abstract}
Resumo
Os anéis de crescimento de Cedrela fissilis foram analisados em duas unidades fitogeográficas do estado do Paraná, em áreas de Florestas Ombrófilas Densa e Mista, litoral e planalto, no domínio da Floresta Atlântica, buscando encontrar sinais climáticos nas séries radiais temporais de anéis de crescimento. As áreas não apresentam estação seca bem definida, com precipitações bem distribuídas ao longo do ano. $\mathrm{Na}$ área do litoral, as temperaturas são altas e constantes durante todo o ano, enquanto que na área do planalto as temperaturas são mais amenas, ocorrendo considerável variação durante o ano. Nas duas áreas foram coletadas 3-4 amostras radiais dos indivíduos selecionados, sendo 15 no litoral e 21 no planalto. Os anéis de crescimento foram marcados, mensurados, submetidos ao controle de qualidade por meio do programa COFECHA ${ }^{\circledR}$ e as cronologias foram geradas utilizando o programa ARSTAN $^{\circledR}$. Sinais climáticos nas séries foram identificados utilizando-se Correlações de Pearson. As séries analisadas provenientes da área de planalto apresentaram melhores correlações com as variáveis temperatura e precipitação, sendo identificados sinais climáticos, enquanto que as séries do litoral não puderam ser datadas com total acurácia. É atribuída a hipótese da competição, principalmente por luz, para explicar a dificuldade na datação dos indivíduos do litoral.

Palavras-chave: Anéis de crescimento; dendrocronologia; Floresta Atlântica.
\end{abstract}

\begin{abstract}
Climatic signals in growth rings of Cedrela fissilis in different types of South Brazil rainforests. Cedrela fissilis tree-rings were examined in two distinct phytogeographic units of the State of Paraná, Brazil. The analysis was carried out in areas of tropical rain forest, Atlantic Forest (cost), and Araucaria Forest (plateau) in order to seek for climatic signals in tree rings series. In the coast area, temperatures are high and constant throughout the year, while in the area of the plateau, temperatures are milder and considerable variations occur during the year. In both areas 3-4 radial samples of each selected individual were collected, 15 from the coast and 21 from the plateau. Growth rings were marked, measured, submitted to quality control using the COFECHA ${ }^{\circledR}$ software, and chronologies generated using the ARSTAN $^{\circledR}$ software. Climatic signals in growth rings were identified using Pearson correlations. Tree rings series from plateau revealed better correlations with temperature and rainfall compared to the coast ones; they were accurately dated while the other from coast could not be dated with accuracy. Climatic signals were found in the well-dated chronology. The hypothesis of competition, mainly by light, is raised to explain the difficulty to establish a good chronology for coast area.
\end{abstract}

Keywords: Growth rings; dendrochronology; Atlantic Forest.

\section{INTRODUÇÃO}

A dendrocronologia é uma ciência que tem sido mais amplamente utilizada em regiões de climas temperados e áridos, com aplicações nas mais diversas áreas do conhecimento, como ecologia, climatologia, geologia e antropologia, entre outras (SCHWEINGRUBER, 1996). Essa ciência baseia-se, principalmente, no fato de que as plantas que crescem em ambientes regidos por forte sazonalidade climática apresentam ciclos anuais de crescimento, podendo, em muitas espécies lenhosas, causar 
demarcações no xilema secundário, denominadas anéis de crescimento (FRITTS, 1976; SCHWEINGRUBER, 1989).

Inicialmente, acreditava-se que, em regiões tropicais e subtropicais, devido à menor sazonalidade climática e à aparente falta de uma condição limitante de crescimento, as árvores desses ambientes crescessem continuamente durante o ano, não formando, portanto, anéis de crescimento anuais (LIEBERMAN et al., 1985). Devido aos esforços empreendidos por vários pesquisadores, como apresenta Worbes (2002), essa ideia foi contestada, pois significativo número de espécies tropicais e subtropicais, crescendo em determinadas condições, apresentam anéis de crescimento anuais, sendo esse fenômeno comprovado por meio de observações anatômicas do lenho e fenológicas, além da aplicação de uma série de metodologias, como a utilização de faixas dendrométricas, marcações cambiais, densitometria de raios $\mathrm{X}$ e datações-cruzada, entre outros.

Apesar de serem relativamente recentes e ainda fragmentados, os estudos relacionados à dinâmica de crescimento de espécies tropicais e subtropicais vêm elucidando o entendimento da formação dos anéis de crescimento nessas regiões, sendo hoje reconhecida uma vasta lista de espécies arbóreas com reconhecida formação anual de anéis de crescimento, além do reconhecimento de uma série de fatores responsáveis por essa formação de caráter anual dos mesmos.

A aplicação da dendrocronologia nos trópicos tem demonstrado grande potencial (i) na reconstrução histórica das condições de crescimento das árvores, (ii) no melhor entendimento das respostas fisiológicas dos indivíduos frente às mudanças das condições ambientais e (iii) na construção e validação de modelos de crescimento, entre outros usos (ROZENDAAL; ZUIDEMA, 2011). Entretanto, é completamente aceito que a aplicação da dendrocronologia nos trópicos se dá de forma muito mais difícil e criteriosa do que com espécies provenientes de zonas temperadas. Diversos fatores, como a dificuldade de visualização dos anéis de crescimento (JACOBY, 1989), a escassez de árvores longevas (WORBES, 1999), a impossibilidade da utilização de indivíduos mortos, devido à alta taxa de decomposição da madeira morta (BULTMAN; SOUTHWELL, 1976), a grande frequência de anéis falsos e faltantes, devido a fatores exógenos (FAHN et al., 1981) e a diversidade de respostas das diferentes espécies frente as mesmas condições ambientais (PALLARDY; KOZLOWSKI, 2008), apresentam-se como obstáculos e/ou fatores limitantes à aplicação dessa Ciência. Nesse contexto, Worbes (1989) atenta sobre a importância da seleção de espécies potenciais para esse tipo de estudo. Tomazello-Filho et al. (2000) apresentam uma série de características, demonstrando o potencial dendrocronológico para algumas espécies pertencentes a família Meliaceae, como (i) o significativo número de espécies nos ecossistemas florestais, com ampla diversidade ecológica em vários continentes, (ii) apresentação de eventos fenológicos distintos, como a queda de folhas na estação seca, em condições naturais e de plantio, (iii) madeira com estrutura anatômica característica, pela nitidez e delimitação dos anéis de crescimento, (iv) árvores com altas taxas de crescimento em diâmetro do tronco e altura, (v) árvores de densidade média, favorecendo a coleta de amostras de madeira por métodos não destrutivos, (vi) disponibilidade de informações sobre a taxa de crescimento, pela marcação do câmbio, uso de bandas dendrométricas e medição da largura dos anéis anuais de crescimento, (vii) extensa bibliografia sobre a taxonomia, dendrologia, botânica, florística, fitossociologia, silvicultura, propriedades da madeira etc.

Entre as espécies da Família Meliaceae, Cedrela fissilis Vell., popularmente conhecida como cedro-rosa, apresenta grande amplitude ecológica, ocorrendo desde a Costa Rica, incluindo o Panamá, Bolívia, Colômbia, Equador, Paraguai, Peru, Uruguai e Venezuela, até o sul do Brasil e nordeste da Argentina (GONZÁLES, 1976; PENNINGTON et al., 1981; GARTLAND et al., 1996). É uma espécie decídua, perdendo completamente suas folhas na fase de maturação dos frutos, com período de caducifólia variando em função das condições climáticas. Devido às ótimas propriedades de sua madeira, como, por exemplo, alta durabilidade quando exposta ao tempo e facilidade em se trabalhar, o cedro possui grande importância econômica, sendo muito utilizado na indústria madeireira, ocupando o quarto lugar dentre as espécies brasileiras madeireiras mais exportadas (ANGELO et al., 2001). É uma árvore largamente empregada no paisagismo de parques e grandes jardins e recomendada na composição de reflorestamentos heterogêneos de áreas degradadas de preservação permanente (LORENZI, 1992).

Em estudos prévios, C. fissilis apresentou madeira com características anatômicas distintas e anéis de crescimento anuais de fácil visualização, caracterizados pelo parênquima marginal e porosidade semiporosa (MARCATTI et al., 2006). O gênero Cedrela tem demonstrado potencial em aplicações dendrocronológicas, permitindo a construção de cronologias, a detecção de sinais climáticos em seus anéis de crescimento e a projeção de ciclos de cortes silviculturais, entre outras (DÜNISH, 2005; 
BRIENEN; ZUIDEMA, 2006; IWASKI-MAROCHI, 2007). Boninsegna et al. (1989) determinaram, com acurácia, a idade e a taxa de crescimento de Cedrela fissilis, além de outras espécies, em Missiones, Argentina. Dünish et al. (2003), estudando a espécie Cedrela odorata em área de clima tropical do Brasil, verificaram a existência de sinais climáticos na cronologia da espécie, indicando que o crescimento de C. odorata responde significativamente à quantidade de precipitação ocorrida nos meses de março até maio da estação de crescimento anterior. Já Dünish (2005) observou que o crescimento de Cedrela fissilis na área de estudo anterior responde da mesma maneira que $C$. odorata.

As cronologias construídas com C. fissilis, porém localizadas em uma região subtropical, indicam que o crescimento da espécie é mais influenciado pela temperatura de maio do período anterior de crescimento, além da temperatura do mês de novembro da estação de crescimento, não sendo identificadas relações significativas com a precipitação. Brienen e Zuidema (2005), estudando a relação da quantidade de precipitação e a largura dos anéis de crescimento de espécies tropicais na Bolívia, verificaram que o crescimento de $C$. odorata é influenciado positivamente pela precipitação do período de transição (maio e junho) da estação chuvosa para a seca na estação de crescimento do ano anterior. Lobão (2011) verificou um sinal climático comum nas cronologias de largura dos anéis de crescimento de $C$. fissilis e C. odorata em diferentes microssítios da região tropical do Acre, Brasil, indicando que nas condições de crescimento estudadas existe um fator climático específico para a região. Rauber (2010), em uma região subtropical do Brasil, também conseguiu, com acurácia, datar indivíduos de $C$. fissilis por meio dos anéis de crescimento. Além disso, a autora encontrou sinais climáticos na cronologia, indicando que a precipitação do período de dezembro a abril da estação de crescimento anterior explica grande parte da variação de crescimento anual.

A partir do exposto, percebe-se que o gênero Cedrela e a espécie C. fissilis apresentam grande potencial para o desenvolvimento de estudos dendrocronológicos em regiões tropicais e subtropicais, permitindo obter informações relevantes sobre a autoecologia da espécie em uma escala temporal.

O presente trabalho objetivou analisar os anéis de crescimento de indivíduos adultos de $C$. fissilis ocorrendo em diferentes gradientes climáticos e altitudinais pertencentes a distintas unidades fitogeográficas de áreas do domínio da Floresta Atlântica no estado Paraná (primeiro planalto e litoral). Além de buscar datar os indivíduos com acurácia, foram investigados sinais climáticos nos anéis de crescimento da espécie.

\section{MATERIAL E MÉTODOS}

Para este estudo, foram avaliadas 36 árvores de Cedrela fissilis, sendo 15 pertencentes à formação de Floresta Ombrófila Densa Submontana (FODSM) e 21 de Floresta Ombrófila Mista Montana (FOMM) do estado do Paraná, Brasil (Figura 1). Procurou-se selecionar indivíduos adultos e representativos nessas formações, sendo estes aparentemente sadios e com boa formação do tronco e copa.

\section{Áreas de estudo}

Litoral paranaense

Os indivíduos localizam-se em área pertencente à Sociedade de Pesquisa em Vida Selvagem e Educação Ambiental (SPVS), localizada no município de Antonina, Paraná, e inserida no domínio da Área de Proteção Ambiental de Guaraqueçaba (Figura 1). As árvores foram selecionadas em áreas remanescentes de floresta, sem indícios e/ou histórico de ação antrópica recente, em domínio fitogeográfico da Floresta Ombrófila Densa Submontana. Os indivíduos estão localizados em uma parcela permanente situada na altitude de $300 \mathrm{~m}$ a.n.m. Segundo Köppen, o clima da região é subtropical úmido mesotérmico (Cfa), sem estação seca definida e sempre úmido. Dados climáticos médios (Figura 2) para um período de registros de 21 anos (de 1978 a 1999) mostram uma precipitação média anual de $2.778 \mathrm{~mm}$ e temperatura média de $21,4{ }^{\circ} \mathrm{C}$ (IAPAR, 2011).

\section{Primeiro planalto paranaense}

Para representar essa região, foram utilizadas duas áreas atualmente protegidas e localizadas no perímetro urbano da cidade de Curitiba (Bosque da Copel e Bosque do Jardim Botânico), Paraná, com altitude média de 945 m a.n.m (Figura 1). O domínio fitogeográfico das áreas é a Floresta Ombrófila Mista Montana (floresta com araucária). 


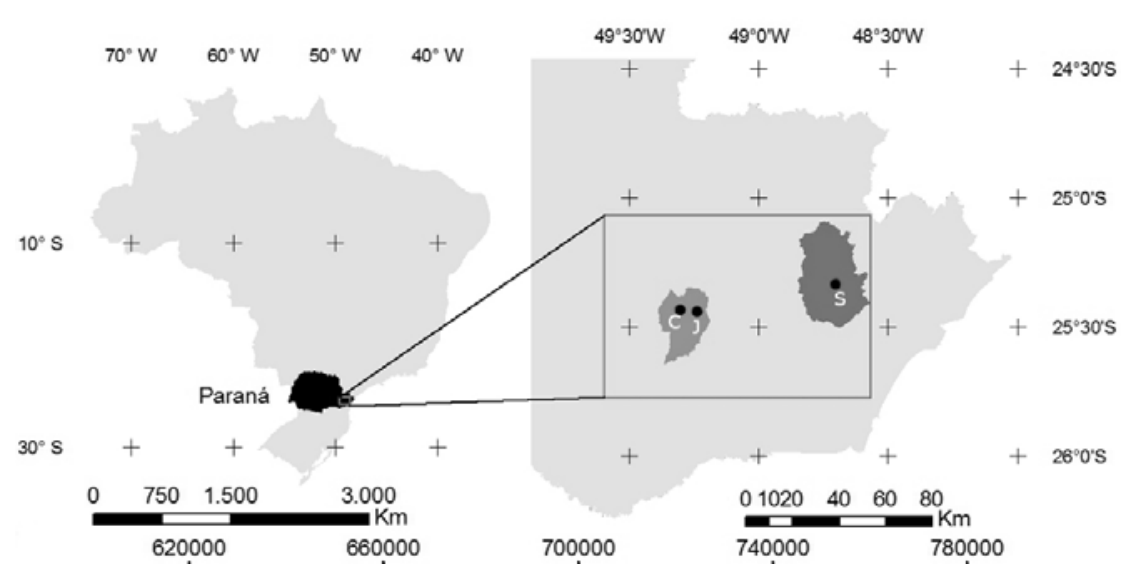

Figura 1. Localização das áreas de estudo nos municípios de Curitiba (C, J) e Antonina (S), Paraná, Brasil. Figure 1. Location of focused areas in the cities of Curitiba (C, J) and Antonina (S), southern Brazil.

Bosque da Copel: situado no bairro Bigorrilho, com área de aproximadamente 5 ha, coberto por vegetação secundária em estágio avançado de regeneração e mantido sob proteção pela Copel desde 1976. O bosque é um remanescente de uma grande extensão de mata de araucária que foi reduzida pelo processo de urbanização e crescimento da cidade. Foram selecionados 9 indivíduos dessa localidade.

Bosque do Jardim Botânico Municipal: localizado no interior das dependências do Jardim Botânico, com área de aproximadamente 7 ha e fechado desde 2006 para entrada de pedestres. Foram selecionados 8 indivíduos dessa área, que é composta por vegetação nativa em regeneração.

A região de Curitiba encontra-se sob o clima subtropical úmido mesotérmico de verões frescos, inverno com geadas frequentes e sem estação seca, sendo considerado como do tipo Cfb na classificação de Köppen. Dados climáticos médios (Figura 2), para um período de registros de 27 anos, mostram precipitação anual de 1.498 mm e temperatura média de 17,3 C (IAPAR, 2011).
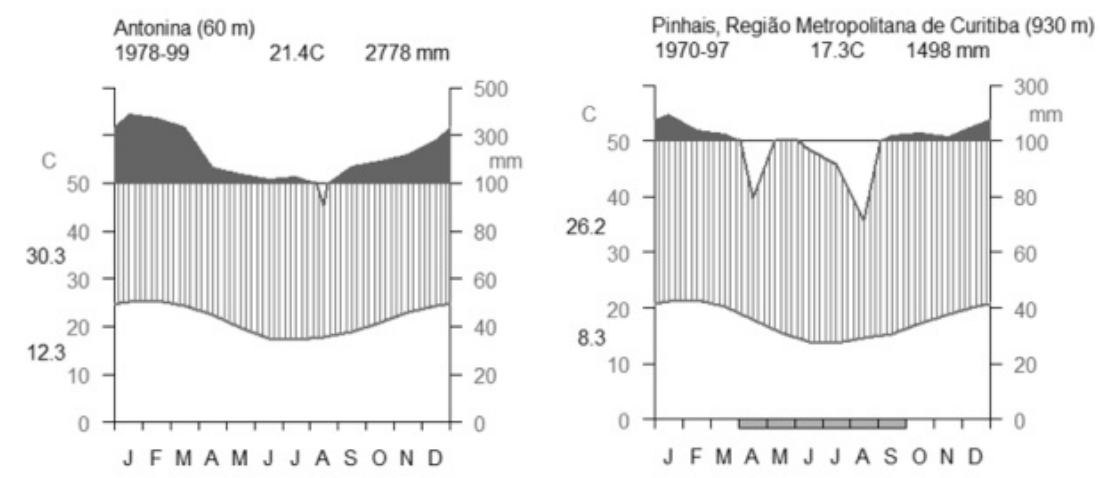

Figura 2. Diagramas climáticos das regiões de Antonina e Pinhais, representando o município de Curitiba, elaborados segundo Walter e Lieth (1967). Modificado de IAPAR (2011).

Figure 2. Climatic diagrams from Antonina and Pinhais, here representing the city of Curitiba, produced according to Walter e Lieth (1967). Modified from IAPAR (2011).

\section{Amostragem e preparo}

Com auxílio da sonda de Pressler (Ø $5 \mathrm{~mm}$ ), foram removidas amostras radiais (3-4 raios) por indivíduo selecionado. As amostras foram fixadas em suportes de madeira, de modo a expor os elementos anatômicos em sentido longitudinal. Posteriormente foram polidas as secções transversais das amostras, com lixas de diferentes gramaturas (de 60 até 600 grãos), proporcionando melhor contraste dos anéis de crescimento e das outras estruturas anatômicas do lenho. As amostras foram digitalizadas utilizando-se 
scanner de mesa com 600 ppp (pontos por polegada) de resolução. Através das imagens obtidas, a largura dos anéis de crescimento inicialmente reconhecidos e delimitados foi medida com auxílio do software de análise de imagens Image Pro Plus ${ }^{\circledR}$ (Media Cybernetics).

\section{Séries cronológicas}

Após mensuração da largura dos anéis de crescimento, foi realizado o controle da qualidade e verificação da sincronização das séries radiais temporais por meio do programa COFECHA ${ }^{\circledR}$ (HOLMES et al., 1986). O programa COFECHA ${ }^{\circledR}$ sobrepõe seguimentos das séries cronológicas com a média construída das demais amostras e verifica estatisticamente a datação, possibilitando identificar possíveis erros devidos à presença de anéis falsos, flutuações intra-anuais ou anéis faltantes. Amostras com baixa correlação foram removidas, de maneira a maximizar o sinal das séries utilizadas para a construção da cronologia.

Para construção das cronologias, foi utilizado o programa ARSTAN ${ }^{\circledR}$ (COOK; HOLMES, 1984). As tendências de crescimento relacionadas aos sinais não climáticos foram removidas, de acordo com Cook e Kairiukstis (1989), por meio da padronização das séries através do ajuste das mensurações originais dos anéis de crescimento com uma função exponencial negativa, seguida da aplicação de uma função spline cúbica, com porcentagem de longitude de série de $67 \%$.

\section{Relações com o clima}

Para estudar a relação entre a precipitação e temperatura e a largura dos anéis de crescimento das árvores, as cronologias foram correlacionadas com dados históricos pluviométricos mensais e de temperatura do ar (máxima, média e mínima) de estações meteorológicas próximas às regiões de estudo, sendo localizadas a aproximadamente $8,5 \mathrm{~km}$ e $15 \mathrm{~km}$ de distância para as áreas de planalto e litoral, respectivamente. Correlações de Pearson foram calculadas, de maneira a testar as relações entre as precipitações anuais, mensais e períodos acumulados de precipitações e temperaturas com o índice da cronologia. Utilizou-se para essa análise até 19 meses, portanto, parte do ano anterior foi incluído, devido a investigações prévias que constataram a influência do ano anterior no crescimento radial de espécies do gênero Cedrela (DÜNISCH et al., 2003; DÜNISCH, 2005; BRIENEN; ZUIDEMA, 2005). Dados climáticos históricos dos municípios de Pinhais, representando a Região Metropolitana de Curitiba (primeiro planalto), e de Antonina (litoral) foram obtidos através do Sistema Meteorológico do Paraná (SIMEPAR).

\section{RESULTADOS E DISCUSSÃO}

\section{Datação cruzada}

Por meio do controle da qualidade da largura dos anéis de crescimento, de um total de 71 séries radiais provenientes de 20 árvores da área do planalto, aproveitaram-se 22 séries de 11 indivíduos para compor a cronologia com correlação significativa de 0,566 , demonstrando um sinal comum para as 11 árvores. Para o litoral, a cronologia foi composta por 38,5\% das séries radiais amostradas e pertencentes a 11 árvores, sendo que a cronologia não alcançou o ponto crítico estabelecido pelo programa COFECHA ${ }^{\circledR}$ $(0,541)$, ficando em 0,356 .

A exclusão de indivíduos e de séries radiais com baixa correlação com os demais é comum para o gênero Cedrela, além de outras espécies tropicas, sendo que esse fato pode ser observado em trabalhos conduzidos por Dünisch et al. (2003), Rauber (2010) e Lobão (2011).

No planalto, a idade dos indivíduos variou de 12 a 22 anos, enquanto que no litoral essa variação foi maior, 12 a 34 anos. A alta correlação dos indivíduos de Cedrela no primeiro planalto pode ser explicada pela homogeneidade de tamanho das séries temporais, sendo que os indivíduos mais longevos foram excluídos, devido à sua baixa correlação ou pela impossibilidade de datação dos anéis devido à presença frequente de sinais de podridão no interior do tronco. Outro fator importante a ser considerado é a posição sociológica comum dos indivíduos, dominantes na floresta, disputando luz com apenas alguns indivíduos isolados de Araucaria angustifolia que se destacam na paisagem. A análise de componentes principais, realizada pelo programa ARSTAN, indicou que os primeiros componentes das cronologias Arstan e Residual explicaram 55,24\% e 55,75\% da variância total do crescimento anual nos últimos 19 anos para os indivíduos do planalto. Devido às semelhanças encontradas no padrão de crescimento dos indivíduos do planalto, os dados dos dois locais de coleta foram agrupados em uma única cronologia. 
Em contraste, a baixa correlação das séries dos indivíduos do litoral poderia ser atribuída à competição interespecífica por luz, diferença de idades dos indivíduos amostrados ou mesmo pela dificuldade da correta identificação de anéis falsos ou faltantes, devido à pequena área de observação oferecida pelas amostras (baguetas). Segundo Dünisch et al. (1999), a pressão competitiva de árvores vizinhas muda a direção de crescimento da árvore, podendo causar a formação de anéis pouco conspícuos, resultantes de uma atividade cambial descontínua, dificultando sua identificação e correlação das amostras de árvores em outras situações. Dificuldades de identificação de anéis de crescimento em baguetas também foram observadas por Stahle et al. (1999) para Pterocarpus angolensis DC. no Zimbábue. Os autores afirmam que a acurácia da datação poderia ser melhorada com uma maior área de observação, de, no mínimo, Ø 5 cm, permitindo assim evidenciar flutuações anatômicas não anuais.

Estatísticas descritivas e a representação gráfica das cronologias são apresentadas na (Tabela 1) e (Figura 3), respectivamente.

Tabela 1. Descrição estatística da datação-cruzada dos anéis de crescimento dos indivíduos de C. fissilis em estudo.

Table 1. Statistical description of crossdating growth rings from focused individuals of C. fissilis.

\begin{tabular}{lcc}
\hline \multirow{2}{*}{ Descrição } & \multicolumn{2}{c}{ Área } \\
\cline { 2 - 3 } & Planalto & Litoral \\
\hline Série codatada & $1985-2009$ & $1972-2010$ \\
Número de séries datadas & 22 & 15 \\
Indivíduos & 11 & 11 \\
Total de anéis em todas as amostras & 452 & 365 \\
Total de anéis checados & 451 & 364 \\
Correlação entre as séries & 0,566 & 0,313 \\
Sensibilidade média & 0,49 & 0,471 \\
Comprimento médio das séries & 20,5 & 24,3 \\
\hline
\end{tabular}

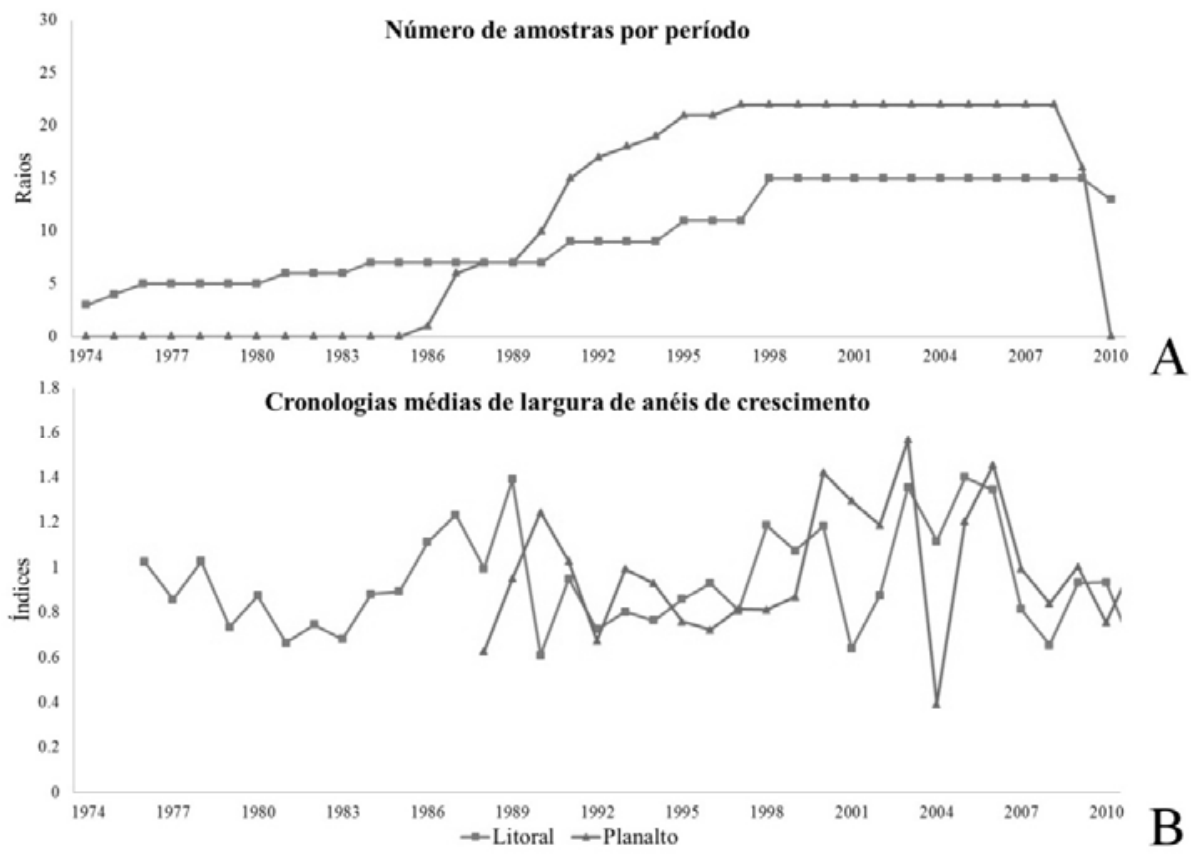

Figura 3. Cronologias Arstan e o número de amostras do lenho utilizadas em suas construções.

Figure 3. Arstan chronologies and the number of wood samples used in their construction. 


\section{Correlações entre a cronologia e o clima}

A identificação de correlações entre cronologias e variáveis climáticas permite a reconstrução de climas passados, sendo uma das principais aplicações da dendrocronologia (SPEER, 2010). Entretanto, para tal aplicação, é necessária uma cronologia robusta, obtida com árvores que apresentem um sinal comum de crescimento. No presente estudo são apresentadas correlações entre precipitação e temperatura (Figura 4) para a cronologia desenvolvida com as árvores do planalto, tendo em vista a existência do sinal comum entre elas, demonstrado através de sua correlação significativa (Tabela 1). Não são apresentadas correlações entre a cronologia e clima para o litoral, já que não foi possível identificar um sinal comum nas árvores e, portanto, tais correlações seriam apenas especulativas.

A análise de correlação entre precipitação e largura dos anéis de crescimento de C. fissilis mostra que a diferença de disponibilidade de água durante os anos explica, pelo menos, uma porção da variação do crescimento anual encontrado na espécie (Figura 4). A correlação positiva entre a precipitação da estação de crescimento anterior indica que o suprimento de água influencia, especialmente, na formação de reservas de nutrientes, que por sua vez são mobilizadas no início do próximo período de crescimento (HÖLL, 1985). Dünish et al. (2003) demonstraram que o crescimento radial nos dois primeiros meses após o período de dormência em Cedrela dá-se em decorrência da mobilização da reserva de nutrientes. Esse comportamento já foi evidenciado em outros trabalhos para o gênero Cedrela (BRIENEN; ZUIDEMA, 2005; RAUBER, 2010; LOBÃO, 2011).

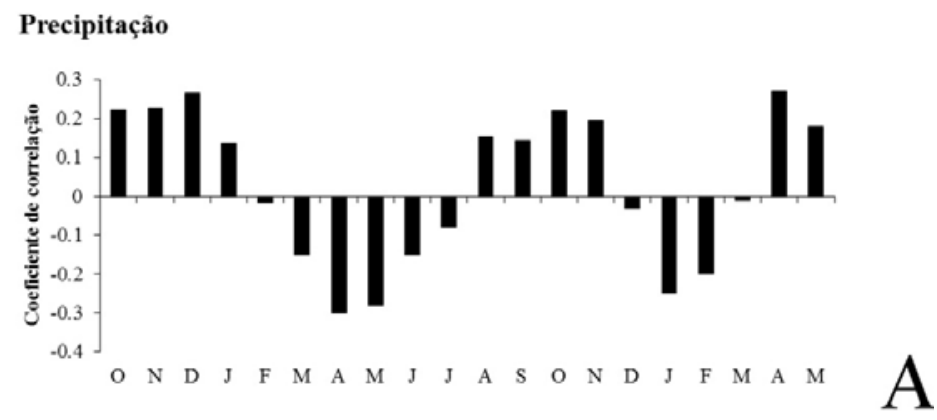

Temperatura mínima

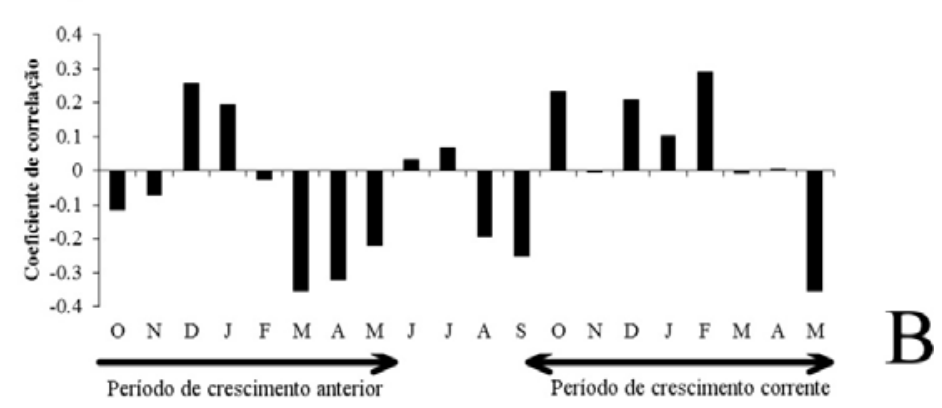

Figura 4. Correlações entre a largura dos anéis de crescimento de $C$. fissilis e precipitação média mensal e temperatura mínima média mensal. Setas identificam os períodos de crescimento do ano anterior e corrente. Para precipitação (A), os meses ao longo do eixo x referem-se ao mês central de um período acumulado de 3 meses, por exemplo: o coeficiente de correlação para o mês $\mathrm{N}$ é aquele entre o índice de crescimento corrente e a precipitação total de outubro até dezembro. Para temperatura (B), as correlações são entre o índice de crescimento corrente e o mês corrente.

Figure 4. Correlations between the width of growth rings of $C$. fissilis and mean monthly rainfall and mean monthly minimum temperature. Arrows identify growth periods of current and previous year. For precipitation (A), the months on the $\mathrm{x}$-axis refer to the central month of period of 3 months accumulated precipitation, for example: the correlation coefficient for month $\mathrm{N}$ is the one between the total precipitation from October to December and the current growth index. For temperature (B), the correlations are between the current growth index and current month minimum temperature. 
Também foram encontradas correlações positivas no início da estação de crescimento, coincidente com o período de altas temperaturas e com maiores horas de luminosidade diária. As árvores utilizadas para compor a cronologia são jovens (Tabela 1) e dividem o extrato superior da floresta apenas com poucos indivíduos de A. angustifolia (Bertol.) Kuntze. Portanto, provavelmente utilizam o aporte hídrico e altas temperaturas nos meses iniciais da estação de crescimento na produção de biomassa, refletida na largura dos anéis de crescimento. A água no início da estação de crescimento é fundamental para que a espécie, no período de produção de novas folhas e flores, tenha maiores condições de aumento de biomassa.

Os padrões de correlações para as temperaturas mínimas e a largura dos anéis de crescimento encontrados corroboram aqueles encontrados por Rauber (2010). Altas temperaturas mínimas no período de crescimento anterior, assim como altas temperaturas mínimas no período de crescimento corrente, influenciam positivamente na largura dos anéis de crescimento de C. fissilis (Figura 4). A região de estudo apresenta verões frescos, com considerável diminuição da temperatura em alguns dias, principalmente durante a noite. Portanto, condições com baixas temperaturas podem diminuir o metabolismo de $C$. fissilis e altas temperaturas mínimas no verão teriam efeitos positivos no crescimento da espécie.

Correlações negativas para precipitação são observadas no período correspondente ao final da estação de crescimento anterior até o mês de julho. Em eventos de elevada precipitação durante algum período do ano, a saturação hídrica do solo poderá influenciar negativamente os processos fisiológicos relacionados ao crescimento de $C$. fissilis. Aliado a isso, também são encontradas correlações negativas para as temperaturas mínimas em período concomitante (março-maio). Esse resultado indica que, na ausência de elevadas temperaturas durante esse período, o excesso de água permanecerá por mais tempo no solo, deixando os indivíduos sob uma ação prolongada de estresse.

\section{CONCLUSÕES}

- Os resultados obtidos confirmam a potencialidade da espécie C. fissilis para estudos dendrocronológicos em regiões subtropicais.

- A acurácia de datação dos indivíduos pode ser dificultada por fatores relacionados à estrutura da floresta.

- Para os indivíduos estudados na região do litoral, amostras com uma maior área de observação e o controle das amostras com alguns discos completos de madeira poderiam melhorar a qualidade da cronologia.

- Outro fator a ser refletido é a seleção mais rigorosa dos indivíduos, procurando-se optar por árvores que apresentem mesma condição de copa, proximidade a cursos d'água, trilhas e semelhantes condições de solo, entre outros aspectos, de maneira a minimizar fatores que eventualmente poderiam gerar "ruídos" e encobririam os sinais climáticos.

- $\quad$ Em florestas maduras, a ocorrência de C. fissilis está intimamente ligada às bordas e clareiras, sendo escassos os indivíduos de grande porte no interior das mesmas. Sendo assim, a escolha rigorosa dos espécimes poderia ter influências diretas na quantidade de amostras por área.

- As árvores estudadas no planalto, por estarem em condições mais homogêneas de suprimento de água e luz, apresentaram melhores correlações e compuseram uma cronologia com sinal climático comum. Contudo, por ser uma cronologia pequena, com poucos anos analisados, as relações entre clima e crescimento encontradas não devem ser generalizadas para a espécie, mas entendidas como caracterização da população estudada.

\section{REFERÊNCIAS}

ANGELO, H.; BRASIL, A. A.; SANTOS, J. Madeiras tropicais: análise econômica das principais espécies florestais exportadas. Acta Amazônica, Manaus, v. 31, n. 2, p. 237 - 248, 2001.

BONINSEGNA, J. A.; VILLALBA, R.; AMARILLA, L.; OCAMPO, J. Studies on the tree rings, growth rates and age-size relationships of tropical tree species in Missiones, Argentina. IAWA Bulletin, Utrecht, v. 10, n. 2, p. 161 - 169, 1989. 
BRIENEN, R. J. W.; ZUIDEMA, P. A. The use of tree rings in tropical forest management: projecting timber yields of four Bolivian tree species. Forest Ecology and Management, Amsterdam, v. 226, p. 256 26, 2006.

Relating tree growth to rainfall in Bolivian rain forests: a test for six species using tree ring analysis. Oecologia, New York, v. 146, p. 1 - 12, 2005.

BULTMAN, J. D.; SOUTHWELL, C. R. Natural resistance of tropical American woods to terrestrial wooddestroying organisms. Biotropica, Lawrence, v. 8, n. 2, p. 71 - 95, 1976.

COOK, E.; KAIRIUKSTIS, L. Methods of dendrochronology: applications in the environmental sciences. Dordrecht: Kluwer Academic, 1989.

COOK, E. R.; HOLMES, R. L. Program ARSTAN users manual. Tucson: The University of Arizona Press, 1984.

DÜNISCH, O.; SCHROTH, G.; MORAIS, R. M.; EMBREICH, M. Water supply of Swietenia macrophylla King and Carapa guianensis Aubl. in three plantation systems. In: BAUCH, J.; DÜNISCH, O.; GASPAROTTO, L. Investigations on tree species suitable for the recultivation of degraded land areas in Central Amazonia. Hamburg: Bundesforschumsanstalt fur Forst-und Holzwirtschaft, 1999. p. 29 - 46.

DÜNISCH, O.; MONTÓIA, V. R.; BAUCH, J. Dendroecological investigations on Swietenia macrophylla King and Cedrela odorata L. (Meliaceae) in the Central Amazon. Trees - Structure and Function, Heidelberg, v. 17, n. 3, p. 244 - 250, 2003.

DÜNISCH, O. Influence of the El Niño southern oscillation on cambial growth of Cedrela fissilis Vell. in tropical and subtropical Brazil. Journal of Applied Botany and Food Quality, Hamburg, v. 79, n. 1, p. 5 11, 2005.

FAHN, A.; BURLEY, J.; LONGMAN, K. A.; MARIAUX, A.; TOMLINSON, P. B. Possible contributions of wood anatomy to the determination of the age of tropical trees. In: BORMANN, F. H.; BERLYN, G. Age and growth rate of tropical trees: new directions for research. New Haven: Yale University, 1981. p. 31 - 54.

FRITTS, H. C. Tree rings and climate. California: Academic Press, 1976. 567 p.

GARTLAND, H. M.; VOGEL, H.; BOHREN, A. V.; GRANCE, L. A.; CABRAL, J. Ficha técnica de árboles de Missiones: Cedrela fissilis Vell. Aspectos dendrológicos. Yvyraretá, Eldorado, v. 7, n. 7, p. 38 - 41, 1996.

GONZÁLES T. G. E. Propiedades de la madera de algunas meliáceaes de la America tropical. Turrialba, San José, v. 3, p. 8 - 13, 1976.

HÖLL W. Seasonal fluctuation of reserve materials in the trunk wood of spruce (Picea abies (L.) Karst.). Journal Plant Physiology, Stuttgart, v. 117, p. 355 - 362, 1985.

HOLMES, R. L.; ADAMS, R. K.; FRITTS, H. C. Quality control of crossdating and measuring: a user's manual for program COFECHA. In: HOLMES, R. L.; ADAMS, R. K.; FRITTS, H. C. Tree-ring chronologies of Western North America: California, eastern Oregon and northern Great Basin. Tucson: The University of Arizona Press, 1986.

INSTITUTO AGRONÔMICO DO PARANÁ (IAPAR). Médias históricas em estações do IAPAR. Disponível em: <http://www.iapar.br/modules/conteudo/conteudo.php?conteudo=1070>. Acesso em: 01/10/2011.

IWASAKI-MAROCHI, C. Anéis anuais de crescimento de Cedro (Cedrela fissilis - Meliaceae) aplicados à avaliação da taxa de crescimento e dendroclimatologia. $140 \mathrm{f}$. Tese (Doutorado em Engenharia Florestal, Setor de Ciências Agrárias) - Universidade Federal do Paraná, Curitiba, 2007.

JACOBY, G. C. Overview of tree-ring analysis in tropical regions. IAWA Bulletin, Utrecht, v. 10, n. 2, p. 99 - 108, 1989.

LIEBERMAN, D.; LIEBERMAN, M.; PERALTA, R.; HARTSHORN, G. S. Mortality Patterns and Stand Turnover Rates in a Wet Tropical Forest in Costa Rica. Journal of Ecology, London, v. 73, n. 3, p. 915 924, 1985. 
LOBÃO, M. S. Dendrocronologia, fenologia, atividade cambial e qualidade do lenho de árvores de Cedrela odorata L., Cedrela fissilisVell. e Schizolobium parahyba var. amazonicum Hub. Ex Ducke, no estado do Acre, Brasil. 209 f. Tese (Doutorado em Ciências, Área de concentração em Recursos Florestais, com opção em Tecnologia de Produtos Florestais) - Escola Superior de Agricultura "Luiz de Queiroz", Piracicaba, 2011.

LORENZI, H. Árvores brasileiras: manual de identificação e cultivo de plantas arbóreas nativas do Brasil. Nova Odessa, Plantarum, 1992. 368 p.

MARCATI, C. R.; ANGYALOSSY, V.; EVERT, R. F. Seasonal variation in wood formation of Cedrela fissilis (Meliaceae). IAWA Journal, Leiden, v. 27, n. 2, p. 199 - 211, 2006.

PALLARDY, S. G.; KOZLOWSKI, T. T. 3. ed. Physiology of Woody Plants. Burlington: Academic press, 2008. 464 p.

PENNINGTON, T. D.; STYLES, B. D.; TAYLOR, D. A. Meliaceae. Flora Neotropica Monograph, New York, v. 28. p. 235 - 244, 1981.

RAUBER, C. R. Dendroecologia de Cedrela fissilis Vell. (Meliaceae) em um ecótono de Florestas Subtropicais Montanas no Brasil. 80 f. Dissertação (Mestrado em Ciências, Programa de Pós-Graduação em Ecologia) - Universidade Federal do Rio Grande do Sul, Porto Alegre, 2010.

ROZENDAALL, D. M. A.; ZUIDEMA, P. A. Dendroecology in the tropics: a review. Trees - Structure and Function, Heidelberg, v. 25, n. 1, 2011, p. 3 - 16, 2011.

SCHWEINGRUBER, F. H. Tree rings and environment: Dendroecology. Berne: Paul Haupt, 1996. 609 p.

Tree rings: basics and applications of dendrochronology. Doredrecht: Kluwer Academic Publishers, 1989. 290 p.

SPEER, J. H. Fundamentals of tree-rings research. Tucson: The University of Arizona Press, 2010. 368 p.

STAHLE, D. W.; MUSHOVE, P. T.; CLEAVELAND M. K.; ROIG, F.; HAYNES, G. A. Management implications of annual growth rings in Pterocarpus angolensis from Zimbabwe. Forest Ecology and Management, Amsterdam, v. 124, p. 217 - 229, 1999.

TOMAZELlO FILHO, M.; BOTOSSO, P. C.; LISI, C. S. Potencialidade da família Meliaceae para dendrocronologia em regiões tropicais e subtropicais. In: ROIG, F. A. Dendrocronología en América Latina. Mendoza: EDIUNC, 2000. p. 381 - 431.

WORBES, M. Annual growth rings, rainfall - dependent growth and long term growth patterns of tropical trees from the Caparo Forest Reserve in Venezuela. Journal of Ecology, London, v. 87, n. 3, p. 391 - 403, 1999.

WORBES, M. Growth rings, increment and age of trees in inundation forests, savannas and a mountain forest in the neotropics. IAWA Bulletin, Utrecht, v. 10, n.1, p. 109 - 122, 1989.

WORBES, M. One hundred years of tree-ring research in the tropics - a brief history and an outlook to future challenges. Dendrochronologia, München, v. 20, n. 1-2, p. 217 - 231, 2002. 